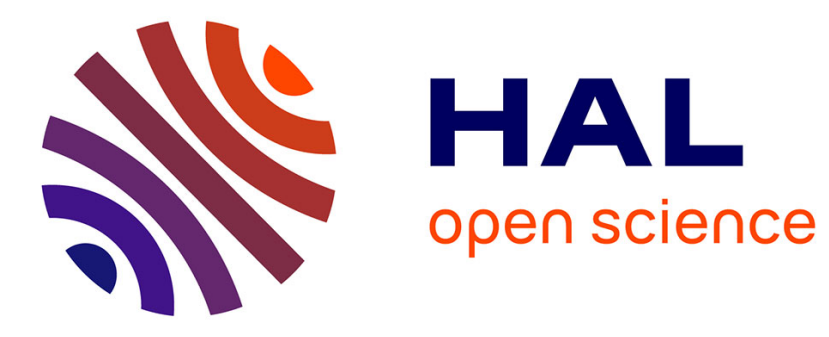

\title{
Microtubule-associated proteins: structuring the cytoskeleton
}

Satish Bodakuntla, A.S. .Jijumon, Cristopher Villablanca, Christian Gonzalez-Billault, Carsten Janke

\section{To cite this version:}

Satish Bodakuntla, A.S. .Jijumon, Cristopher Villablanca, Christian Gonzalez-Billault, Carsten Janke. Microtubule-associated proteins: structuring the cytoskeleton. Trends in Cell Biology, 2019, 10.1016/j.tcb.2019.07.004 . hal-02391963

\section{HAL Id: hal-02391963 https://hal.science/hal-02391963}

Submitted on 3 Dec 2019

HAL is a multi-disciplinary open access archive for the deposit and dissemination of scientific research documents, whether they are published or not. The documents may come from teaching and research institutions in France or abroad, or from public or private research centers.
L'archive ouverte pluridisciplinaire HAL, est destinée au dépôt et à la diffusion de documents scientifiques de niveau recherche, publiés ou non, émanant des établissements d'enseignement et de recherche français ou étrangers, des laboratoires publics ou privés. 


\section{Microtubule-associated proteins: structuring the cytoskeleton}

Satish Bodakuntla ${ }^{1,2}$, Jijumon A.S. ${ }^{1,2}$, Cristopher Villablanca ${ }^{3,4}$, Christian GonzalezBillault ${ }^{3,4, *}$, Carsten Janke ${ }^{1,2, *}$

${ }^{1}$ Institut Curie, PSL Research University, CNRS UMR3348, F-91405 Orsay, France

${ }^{2}$ Université Paris Sud, Université Paris-Saclay, CNRS UMR3348, F-91405 Orsay, France

${ }^{3}$ Center for Geroscience, Brain Health and Metabolism (GERO), Santiago, Chile

${ }^{4}$ Department of Biology, Faculty of Sciences, University of Chile, Santiago, Chile

*Correspondence: chrgonza@uchile.cl (C. Gonzalez-Billault); Carsten.Janke@curie.fr (C.Janke)

Christian Gonzalez-Billault, Department of Biology, Faculty of Sciences, Universidad de Chile, Las Palmeras 3425, 7800024 Santiago, Chile

Telephone: +56229787442

Carsten Janke, Institut Curie, Centre Universitaire, Bâtiment 110, F-91405 Orsay Cedex, France

Telephone: +33169863127 


\begin{abstract}
:
Microtubule-associated proteins (MAPs) were initially discovered as proteins that bind and stabilise microtubules. Today, an ever-growing number of MAPs reveals a more complex picture of these proteins as organisers of the microtubule cytoskeleton with a large variety of functions. MAPs enable microtubules to participate in a plethora of cellular processes such as the assembly of mitotic and meiotic spindles, neuronal development, or the formation of the ciliary axoneme. While some subgroups of MAPs have been exhaustively characterised, a strikingly large number of MAPs remains barely characterized, other than their interactions with microtubules. Here, we provide a comprehensive view on the currently known MAPs in mammals. We discuss their molecular mechanisms and functions, as well as their physiological role and links to pathologies.
\end{abstract}




\section{Defining Structural MAPs.}

Microtubules are a major component of the eukaryotic cytoskeleton that are uniformly assembled from conserved $\alpha / \beta$-tubulin heterodimers. Microtubules play important roles in virtually every cellular process, such as cell division, cell motility, intracellular organization and trafficking of organelles. To fulfil these divergent functions, microtubules assemble into distinct arrays that are characterized by a defined architecture and dynamics. Formation of these assemblies requires specialized proteins that interact with microtubules - the microtubule-associated proteins (MAPs). Based on their mode of action, MAPs can be classified into (a) motile MAPs or motor proteins that generate forces and movement $[1,2]$, (b) enzymes that break or depolymerize microtubules [3], (c) microtubule nucleators [4], (d) end-binding proteins that specifically associate with plus- or minus-ends of microtubules [5], and (e) the so-called structural MAPs. While the first four categories (a-d) of MAPs are clearly defined by their functions, the latter category (e) is a rather vaguely defined, heterogenous group of proteins that bind, and thus stabilize microtubules, but no systematic view on their functions has so far emerged. Here, we discuss the current understanding of the mechanisms and functions of those structural MAPs. Our review focusses on mammalian MAPs, and we refrain from discussing the extensively-studied posttranslational regulation of MAPs, which has been reviewed in detail elsewhere [6, 7].

\section{Discovery of microtubule-associated proteins}

Early efforts to purify tubulin from brain extracts using cycles of assembly-disassembly led to the identification of the first MAPs, MAP1 and MAP2, as higher-molecular components of microtubule assemblies $[8,9]$. At the same time, a different biochemical purification procedure led to the discovery of tau, another protein factor essential for microtubule assembly [10-12]. In contrast to MAP1 and MAP2, tau protein was of lower molecular weight, strikingly correlated with the impact of those proteins on microtubule assemblies: while MAP2 formed long projections at the microtubule surface that increased the distance between single microtubules, microtubules decorated with the smaller tau protein were packed much denser [13]. It thus appeared that MAPs have the ability to control the structure of microtubule assemblies, hence referred to as 'structural MAPs'.

The development of a method to purify tubulin from HeLa cells was instrumental in the discovery of two novel, non-neuronal, MAPs of $210 \mathrm{kDa}$ and $125 \mathrm{kDa}$ [14]. The 210-kDa MAP was considered a bona fide MAP based on its similarity to previously characterized 
MAPs [15], and was named MAP4 [16]. The 125-kDa protein corresponded to the laterdescribed MAP7 [17, 18].

All MAPs had to this point been identified by their capacity to promote the assembly of tubulin into microtubules. However, a considerable share of microtubules never disassembles when tubulin is purified from brain tissue. A closer biochemical characterization of these cold-stable microtubules led to the discovery of the protein STOP (stable tubule only peptide) as a factor that confers cold-stability to microtubules $[19,20]$. As purified STOP protein behaves like a MAP [21], it was later named MAP6.

Apart from those initially characterized major MAPs, several minor proteins were detected in fractions of polymerized tubulin, which all could potentially be MAPs as well [22, 23]. Monoclonal antibody technologies bolstered the purification of some of these potential MAPs, for instance MAP3 [24] and MAP5 [25]. Strikingly, another protein, MAP1C, turned out to be the retrograde motor protein, cytoplasmic dynein [26, 27].

By the end of the 1980s, advances in molecular cloning clarified the identity of many of the MAPs that were so far only biochemically characterized. MAP1 and MAP5 were found to be encoded by distinct genes, but as they are highly similar proteins, were renamed MAP1A and MAP1B [28]. In contrast, different isoforms of MAP2, referred to as MAP2A, 2B and 2C, originate from alternative splicing of the same transcript $[29,30]$. Surprisingly, MAP3 and MAP4, thought to be two distinct proteins based on immunological assays, appeared to be identical gene products, and are now referred to as MAP4 [31]. The cloning of tau protein revealed that a single gene gives rise to six distinct splice isoforms [32,33]. The differential splicing of tau protein received a particularly broad attention, given that tau is the main component of paired helical filaments (PHFs) - one of the pathological hallmarks of Alzheimer's disease [34].

In the 1990s, annotated genome and cDNA sequences allowed the bioinformatic identification of novel MAPs, such as the entire family of mammalian MAPs, the EMAP-like (EML) proteins. EMAPs had initially been discovered as main components of the microtubule cytoskeleton in sea urchin eggs $[35,36]$, and the human homologs of these proteins were found based on sequence similarities $[37,38]$. The fact that the EMAP family has six members in mammals strongly suggests important functions, however so far, little is known. Another technological advance - cDNA libraries - allowed the setup of screens to identify novel MAPs based on their localization to microtubules in cells. This allowed for the identification of epithelial MAP of $115 \mathrm{kDa}$ (E-MAP-115, later named MAP7) [17], GLFND 
[39], and MTR120 (MAP10) [40]. Even screens designed for other purposes could reveal the association of novel proteins with microtubules. For instance, MAP8 was initially discovered as an interaction partner of a protein related to male infertility [41]. Because of its homology to MAP1A/B proteins, MAP8 was also referred to as MAP1S [42]. MAP9, also known as ASAP (Aster-associated protein) was discovered in a screen for proteins involved in intracellular trafficking [43]. Using genome-wide linkage analysis and whole-exome sequencing, mutations of the gene C7orf43 were linked to cases of human microcephaly. C7orf43 encodes a protein that binds microtubules in cells, and was hence named MAP11 [44]. Figure 1 summarizes the timeline of MAPs discovery (Fig. 1).

Finally, advances in proteomics now allow for a more complete identification of proteins that co-purify with microtubules, for instance from Xenopus laevis egg extracts [45]. However, thorough biochemical characterization of newly identified proteins remains an essential prerequisite before validating them as bona fide MAPs (Box 1).

\section{The role of MAPs in structuring the microtubule cytoskeleton}

Since their discovery in the early 1970s, it was clear that MAPs can promote microtubule polymerization, stabilisation and bundling in vitro [10, 46, 47]. When overexpressed in mammalian cells, all MAPs decorated the microtubule cytoskeleton, however only some of them, such as tau, induced microtubule bundling [48, 49]. There have been different hypotheses on how MAPs directly participate in microtubule bundling. MAPs could create a direct physical connection between neighbouring microtubules [50], or alternatively, the stabilisation of microtubules alone could be sufficient to induce bundling [51]. Early studies with the microtubule-stabilising drugs Taxol, or the nonhydrolyzable analogue of GTP, GMPCPP, had already suggested that suppression of microtubule dynamics might be sufficient for the formation of microtubule bundles (discussed in [51]). Indeed, the same appears to be true for MAPs: expression of a minimal microtubule-binding domain of tau, for instance, was sufficient to induce microtubule bundling in vitro [52]. A mechanism by which a minimal MAP domain could promote microtubule bundling was recently proposed based on structural data: upon binding, the positively charged amino-acid patches typically present in MAPs would neutralize the high negative charge of carboxy-terminal tails of tubulins, thus reducing electrostatic repulsion between microtubules, thus allowing spontaneous bundling [53]. A strong argument in favour of this hypothesis is that removal of the negatively charged tubulin tails by subtilisin alone induces microtubule bundling [54]. 
It thus appears that regions of MAPs that do not directly interact with microtubules do not participate in the microtubule bundling. On the contrary, they might even prevent it by pushing microtubules apart from each other. For example, overexpressed full-length MAP4, which has a long projection domain (Fig. 2), binds microtubules without inducing their bundling $[48,55]$, while the MAP4 microtubule-binding domain alone does induce bundling [56]. This essentially leads back to some of the initial observations in vitro [13] and in vivo $[57,58]$, which showed that microtubule spacing is regulated by the size of the projection domains of different MAPs, and therefore, longer projection domains would prevent bundling by keeping microtubules apart (Fig. 3A). In this light, the fact that several MAPs express isoforms with projection domains of variable length $[30,33,59]$ suggests a regulatory mechanism for microtubule network organization. At the same time, the extended projection domains could also serve as interaction platforms for other proteins, discussed below. Notwithstanding the mechanisms involved, presence of MAPs reduces microtubule depolymerization frequency as apparent from longer microtubule life times in the presence of depolymerizing drugs $[49,60]$, or from accumulation of posttranslational modifications on MAP-stabilized microtubules [61]. However, how this stabilization is achieved on the molecular level has for a long time remained difficult to decipher due to the absence of structural data, which is mainly attributed to the disordered structure of many MAPs.

Most MAPs do not adapt a defined structure in solution and were thus generally considered to be disordered proteins. Recently, advances in cryo-electron microscopy have allowed to directly visualize how MAPs bind to microtubules [62]. The protein tau forms an extended structure that binds the microtubule surface along a protofilament, thereby spanning both intra-and inter-dimer tubulin interfaces and interacting with three tubulin monomers [63]. Strikingly, tau was found adjacent to the unstructured C-terminal tails of tubulin, thus underpinning the potential importance of these tails [64] and their posttranslational modifications [65] in controlling the tau-microtubule interactions.

It is highly likely that high-resolution structures of other MAPs will follow soon [66], allowing us to understand their functions from a novel, structural perspective. Similarities of the microtubule-binding domains of several MAPs, such as tau, MAP2 and MAP4 will certainly reflect the way these MAPs bind to microtubules.

A large number of MAPs can potentially coexist on a microtubule and a number of different MAP binding sites exist on the microtubule surface. For example, doublecortin (DCX), a MAP particularly abundant in neurons during development, binds microtubules on a different 
site than tau. The DCX binding site is found between adjacent protofilaments, thus allowing this MAP to connect four tubulin molecules, which both strengthens and determines the interprotofilament and tubulin-dimer interactions. As a result, DCX forces microtubules into a 13protofilament configuration (Fig. 3E) [67].

In the light of the emerging structural biology of MAPs, it is important to remember that MAPs are not merely a 'glue' that sticks to the microtubule surface thereby preventing depolymerization, but rather dynamically bind and unbind microtubules as recently shown for tau [68]. Current work even suggests that tau protein does not stabilize microtubules, but rather allows some of them to remain labile in axons $[69,70]$. This echoes earlier observations for MAP1B, which suggested that this protein actually regulates the dynamic fraction of microtubules $[71,72]$.

\section{$\underline{\text { MAPs as crosslinkers of different cytoskeletal components }}$}

Apart from the 25-nm microtubules, eukaryotic cells contain two other principal cytoskeletal fibre networks: 4-nm actin filaments, and $\sim 10-\mathrm{nm}$ intermediate filaments. The interaction of these different cytoskeletal elements is essential for a range of cellular functions, such as directional cell migration [73], or neuronal pathfinding [74]. Today we know a number of MAPs able to connect microtubules to actin and/or intermediate filaments, which are therefore referred to as cytoskeletal crosslinkers (Fig. 3D).

Two major cytoskeletal crosslinkers are the microtubule-actin crosslinking factors 1 and 2 (MACF1, MACF2). MACF1 was initially discovered as actin crosslinking factor 7 (ACF7) [75], and MACF2 as bullous pemphagoid antigen 1 (BPAG1) [76], or dystonin (DST) [77]. Both proteins are bona fide crosslinkers of the microtubule and actin cytoskeletons [78, 79], but they also interact with intermediate filaments [80, 81]. The striking feature of MACF proteins is their large size (MACF1 $\sim 600 \mathrm{kDa}, \mathrm{MACF} 2 \sim 900 \mathrm{kDa}$ ), and both proteins contain amino-terminal actin- and carboxy-terminal microtubule-binding domains, separated by very long spectrin repeat domains that provide physical spacers between the actin and microtubule networks. The dominant localization cue for MACF proteins appears to be the microtubule network [79], which is why these proteins could primarily be considered as MAPs [82]. Dystrophin, another protein consisting extended spectrin repeats, had initially been described as an actin-binding proteins [83], and only the recent discovery that it can bind microtubules in the muscle cells now qualifies dystrophin as cytoskeletal crosslinker [84]. 
To be able to study the actin-microtubule crosslinking mechanism of MACF1 in vitro, a short version of the protein, called TipAct, was prepared by excluding its long central spectrin domain (Fig. 2), thus encompassing only the amino-terminal actin- and the carboxy-terminal microtubule-binding domains. TipAct tracks microtubule plus-ends in an EB-proteindependent manner, and links them to actin filaments, thus guiding microtubule growth along actin bundles [85].

With their ability to bind three cytoskeletal elements, MACF proteins can participate in a large variety of cellular functions. MACF1 plays a key role in cell migration, thus controlling wound healing $[86,87]$, neuronal migration during brain development $[88,89]$, as well as dendritic arborisation and axon outgrowth [90, 91]. MACF2 is essential for the correct alignment of microtubules and intermediate neurofilaments in large-calibre sensory neurons, which in MACF2-knockout mice are hugely disorganized. This cytoskeletal disorganisation is accompanied by organelle accumulations that reveal defects in axonal transport [80, 92]. Moreover, mutations in MACF2 lead to dystonia musculorum in mice [77] and humans [93]. Alternative splicing of MACF1 and MACF2 can generate variants of the proteins with different domain composition, which can alter their molecular roles in connecting the different cytoskeletal networks. Strikingly, different splice isoforms give rise to distinct biological roles of the proteins, and are also linked to specific pathologies (reviewed in ref. [94-96])

The clear distinction of actin-, intermediate-filament- and microtubule-binding motifs of MACFs makes these proteins prime example of cytoskeletal linkers. Nevertheless, other MAPs have also been implicated in the binding of multiple cytoskeletal components, though their most-studied function relates to their microtubule binding. All members of the MAP1 family, MAP1A, MAP1B and MAP1S, have been shown to interact with actin as well as with microtubules [42, 97-99], with the actin- and microtubule-binding sites clearly separated within the primary sequence of these proteins. It is thus conceivable that these MAPs can serve as cytoskeletal linker proteins and this notion is supported by the observation that MAP1B-deficient neurons display decreased activity of the actin regulators Rac1 and Cdc42 [100].

Finally, a number of reports suggest that other MAPs also interact with different cytoskeletal networks, however the physiological relevance of these mostly biochemically determined interactions still needs to be elucidated (Box 2). 


\section{$\underline{\text { Physiological functions of MAPs }}$}

MAPs help cells in organising the microtubule cytoskeleton (Fig. 3). However, how MAPinduced changes in the cytoskeleton alter the physiological processes is just beginning to be understood. One of the key reasons for this lack of insight is that exogenous expression of MAPs, which was for decades the only way of introducing them into mammalian cells, leads to a variety of artefacts that are most frequently related to an overstabilisation of microtubules. Given that the regulation of microtubule dynamics by stabilisation or destabilisation with MAPs is a highly concentration-dependent biophysical process, any kind of non-physiological expression level can henceforth be artificial. It will thus be key to use gene-targeting approaches to directly label endogenous MAPs to observe their functions at physiological levels.

Still, several insights into cellular functions of MAPs have been gained by overexpression approaches, especially for tau, which is particularly well-explored due to its key role in neurodegenerative disorders (for a detailed review, see [101]). Tau was implicated in intracellular traffic control, as its overexpression [102] and hyperphosphorylation impede transport of synaptic vesicles and organelles in vivo [103, 104]. Strikingly, reduction of tau levels could rescue axonal transport defects observed in mouse models for Alzheimer's disease [105]. It will be important to understand if these effects are due to changes in the structural organisation of the microtubule cytoskeleton, i.e. an alteration in the spacing of axonal microtubules $[57,106]$, or due to a direct regulation of the transport processes by tau protein, as suggested by in vitro reconstitutions of transport in the presence of tau [107, 108]. In a similar manner, other MAPs such as MAP1B [109], MAP2, MAP4 [110, 111], MAP6 [112] and MAP7 [113-116] were shown to influence intracellular transport but perhaps not with the same selectivity (Fig. 3F). Indeed, MAP2, a neuronal MAP that is excluded from axons in differentiated neurons, controls cargo sorting at pre-axonal filtering zone by distinguishing KIF1- from KIF5-dependent cargoes [117]. MAP4 showed a specific inhibition of dynein-driven movements in Xenopus laevis melanocytes, whereas it activated kinesin-2driven transport in these cells [111].

MAPs can also regulate microtubule dynamics by controlling the activity of microtubulesevering enzymes. For instance, MAP4 from Xenopus laevis can inhibit katanin-mediated microtubule severing in vitro [118], and tau, MAP2 and MAP4 can protect microtubules against severing by overexpressed katanin in mammalian cells (Fig. 3C) [119]. 
On the mechanistic side, MAPs might physically impede motors or severing enzymes to interact with microtubules in regions densely decorated with MAPs. The recently discovered tau islands that stall kinesin motility provide a first model for a mechanism that could potentially shield microtubule stretches in cells $[120,121]$. However, there are multiple other mechanisms by which MAPs could influence the function of the microtubule cytoskeleton. Members of the MAP7 family for example directly regulate the motor activity of kinesin-1 $[115,122]$.

MAPs can also control each other. A number of MAPs interact with microtubules at similar sites, and could thus compete for microtubule binding. This has been so far demonstrated for MAP7, which is able to displace tau protein from microtubules in vitro [113]. The competition between tau and MAP7 might play an important role in the determination of neuronal connectivity, as both MAPs are distinctly involved in formation of axonal branches $[123,124]$. These exciting discoveries provide first glimpses at the potentially large variety of regulatory roles of MAPs.

In general, MAPs interact with a plethora of proteins and might thus link the microtubule cytoskeleton to many other cellular functions, such as signal transduction pathways and synaptic functions [125]. For instance, MAP1A/B that interact with postsynaptic density proteins and several ligand-gated ion channels or transmembrane receptors [99, 126], thus controlling synaptic functions. MAP2 associates with a number of protein kinases such as cAMP-dependent protein kinase (PKA) and its regulatory subunit [127], or the tyrosine protein kinases Src and Fyn [128, 129], thus sorting these signalling proteins into the somatodendritic compartment of neurons $[127,130]$. The close link between neuronal, and in particular synaptic signal-transduction machineries and MAPs opens the exciting possibility that neuronal activity could be directly propagated to the microtubule cytoskeleton, and thus regulate its function. Indeed, recent work shows that MACF1 associates with acetylcholine receptors (AChRs) at the neuromuscular synapses and controls the efficiency of signal transmission in mice [131].

MAPs can further interact with membranes, but so far only a few examples are known. The protein tau localizes to the inner side of the plasma membrane [132], which might either titrate this MAP away from the microtubules, or connect microtubules to the plasma membrane (Fig. 3B). MACF1 also associates with the membrane of Golgi vesicles, thus mediating their transport from the trans-Golgi network to the cell periphery [133]. Moreover, the finding that MAP1B regulates the degradation of Rab35 [134] suggests a role in 
membrane trafficking in neurons. It is highly likely that other MAPs also interact with cellular membranes, potentially playing a key role in intracellular integration. Last but not least, MAPs can facilitate viral infections by serving as adapters between the viral particles and the microtubule tracks, on which the virus is transported into the cell nucleus [135].

The multiple interactions of MAPs with functionally important cellular machineries clearly point towards their essential roles in coordinating cytoskeletal functions with a variety of physiological processes. The large size of many MAPs predestines them to be scaffold proteins for entire machineries working on the microtubules, such as transport and signalling complexes. Strikingly, these complexes might even contain tubulin-modifying enzymes [72], thus allowing MAPs to directly regulate the tracks to which they bind.

\section{Implications of MAPs in pathologies}

Given the large number of functions performed by MAPs, it is not surprising that they are also involved in various pathological conditions (reviewed in [136]). Transgenic mice for mutant/missing MAPs have underpinned their importance in maintaining structure and functions of the mammalian brain. For example, mice lacking tau display age-onset neurodegeneration [137]. MAP2- or MAP1B-knockout mice show neurodevelopmental defects [100, 130, 138-140], and mice with disrupted MAP1A gene display Purkinje-cell degeneration [141]. The MAP6-knockout mouse is recognised as a model of schizophrenia $[142,143]$. Strikingly, in all these cases, it is not the pathological accumulation of the MAPs, but their deficiency that leads to the pathology. This underpins their importance in cellular homeostasis, and points toward the possibility that changes in the balance of MAPmicrotubule interactions are sufficient to induce pathological events that lead to neuronal disorders.

This perspective opens up novel prospects for alternative molecular pathways in MAP-related disorders. For example, Alzheimer's and Parkinson's disease, as well as frontotemporal dementia and other tauopathies are characterized by the prevalent pathological hallmark of aggregated tau protein $[34,144,145]$, and research has focussed on tau aggregation as the key event in their pathogenesis. However, it is likely that the initial events are rather early perturbations of the physiological functions of MAPs. Moreover, these initial events could be also regulated by the tubulin posttranslational modifications, proposed to control MAPmicrotubule interactions. For example, recent findings demonstrating that abnormally increased microtubule polyglutamylation leads to neurodegeneration in a variety of neuronal 
cell types in mouse, as well as in a newly discovered human disorder strongly underpin this notion $[146,147]$.

\section{Concluding remarks and future directions: the emerging roles of MAPs}

In this review, we have discussed how 'structural MAPs' fulfil different functions on the microtubule cytoskeleton, beyond their common characteristics - the binding to microtubules. MAPs can regulate microtubule dynamics and organization of microtubules, but also interactions of microtubules with other functionally important proteins such as molecular motors or regulators of microtubule stability. MAPs further link microtubules to other cytoskeletal elements, non-cytoskeletal proteins and protein complexes, or membrane compartments in cells.

Little is known so far about the regulation of local MAP concentrations in cells, which will be one of the crucial questions to answer if the patterning of the microtubule cytoskeleton with MAPs is to be understood. MAPs could recognize specific microtubule configurations, such as parallel or anti-parallel arrays (Fig. 3G). This is well-studied for the proteins TRIM46, a MAP that assembles plus-end-out microtubule arrays in the axon initial segment [148], and PRC1, which accumulates specifically at antiparallel overlapping microtubules at the mitotic spindle midzone $[149,150]$. Another potential regulatory mechanism for MAP localisation could be the tubulin code, i.e. specific tubulin isotypes or posttranslational modifications of microtubules [151], which could attract MAPs to distinct microtubule arrays in cells. In addition, MAPs themselves are posttranslationally modified [6], and might be able to control the posttranslational modifications of the microtubules they bind to [72]. A complex regulatory network coupling the tubulin code to a hypothetical MAP code could thus create a broad variety of microtubule identities that coordinate cytoskeletal functions in cells. Indeed, different MAPs show distinct subcellular localisations in neurons, which correlates with their specific contributions to a variety of neuronal functions [136].

Finally, the concentration of MAPs can be drastically increased by phase transitions. In this process, a protein separates from the surrounding cytosol into a liquid phase droplet of which it is the sole constituent [152]. Droplets of the protein tau could serve as microtubule nucleation centres due to the extraordinarily high concentration of the MAP, which could enrich tubulin above the critical concentration needed for its polymerization [153]. It is tempting to imagine that reversible phase separation takes place on the microtubules, with the 
more tempting possibility that local concentrations needed to initiate phase transition of MAPs are induced by locally enriched tubulin modifications (Fig. 4).

Another emerging concept is that MAPs can impact the molecular structure of microtubules. It has already been shown that DCX can determine the precise number of protofilaments (Fig. 3E) [67], which might explain why in cells microtubules have a much more controlled protofilament number than in-vitro assembled microtubules [154]. Moreover, recent findings suggest that MAPs can generate a whole range of 'unusual' microtubule structures. For example, the protein SSNA1 (a.k.a. NA14), which stabilises single-protofilament protrusions, can generate microtubule branches [155]. Mechanisms of protofilament stabilisation might also allow for the maintenance of damaged microtubules until they are repaired by reincorporation of tubulin dimers (Fig. 4) [156]. Such mechanisms could be rather common in cells, and many MAPs might have such activities depending on their local concentrations [157]. The control of microtubule damage and repair has recently gained an even greater dimension with the discovery that motor proteins damage the microtubule tracks they walk on $[158,159]$. Lattice maintenance might thus be one of the previously neglected cellular functions of MAPs. Moreover, MAPs that bind to the luminal side of microtubules, the socalled microtubule-inner proteins (MIPs), have only recently come into focus [160], and provide novel options for the regulation of microtubule dynamics and structure [161]. Last, but certainly not least, there is an emerging family of proteins that bind to tubulin dimers, called tubulin-associated proteins (TAPs) [162]. While some TAPs might just be MAPs able to interact with soluble tubulin dimers, others could bind exclusively to unpolymerized tubulin dimers, and have a strong impact on microtubule homeostasis in cells, as it was demonstrated for the tubulin-sequestering protein stathmin [163].

In the light of those emerging mechanisms it appears that the name 'structural MAPs', which had been coined for a vaguely defined family of proteins whose only common characteristics is that they bind to microtubules, has gained a greater mechanistic dimension: many of the here-discussed MAPs, and others yet to be identified, might turn out to be the key determinants of cytoskeletal architecture. 


\section{Outstanding questions}

- Does the abundance of MAPs in a cell really reflect their biological importance? What are the functions of less abundant MAPs?

- What could be a novel definition of a MAP, and which experiments/approaches could be used to validate a protein as bona fide MAP?

- How do different MAPs function in a single cell? Do they all have distinct or redundant functions, or do they compete with each other?

- How are local MAP concentrations controlled in cells, and how does this contribute to the patterning of the microtubule cytoskeleton?

- How does the tubulin code, i.e. tubulin isotypes and their posttranslational modifications, control MAP binding to functionally specific microtubules? To what extent do MAPs influence the tubulin code?

\section{$\underline{\text { Acknowledgement }}$}

This work was supported by the ANR-10-IDEX-0001-02 and the LabEx CelTisPhyBio ANR11-LBX-0038. CJ is supported by the Institut Curie, the French National Research Agency (ANR) award ANR-17-CE13-0021, the Institut National du Cancer (INCA) grant 2014-PL BIO-11-ICR-1, and the Fondation pour la Recherche Medicale (FRM) grant DEQ20170336756. SB was supported by the FRM grant FDT201805005465. JAS was supported by the European Union's Horizon 2020 research and innovation programme under the Marie Skłodowska-Curie grant agreement No 675737, and the FRM grant FDT201904008210. SB, CV, CG-B and CJ are supported by the ECOS-CONICYT grant C14B01. CG-B is supported by Fondecyt 1180419 and Fondap 15150012 grants from CONICYT. We are grateful to M Genova, MM Magiera, S Gadadhar (Institut Curie, Orsay, France) for critical reading and instructive discussions of our manuscript. 


\section{$\underline{\text { Figures and Boxes }}$}

\section{Box 1: Redefining MAPs}

Over the years, proteins were discovered to interact with microtubules by various assays.

However, there are a number of difficulties in designing functional experiments to test newly identified proteins for validating their potential role as MAPs. An important question is thus how to define novel proteins as bona-fide MAPs. The early definition of a MAP was that "it copolymerizes with tubulin through repeated cycles of microtubule assembly in vitro; it is not associated with any brain subcellular fraction other than microtubules; in double-label immunofluorescence experiments antibodies against this protein stain the same fibrous elements in cultured cells as are stained by anti-tubulin; and this fibrous staining pattern is dispersed when cytoplasmic microtubules are disrupted by colchicine." [24]. While this definition is somewhat still valid, it might need some refinement to consider potential artefacts, for instance that overexpression of some proteins can force them into nonphysiological microtubule decoration or that overexpression of these MAPs could favour lowaffinity interactions. Therefore, thorough biochemical characterization of newly identified proteins is still an essential prerequisite before considering them MAPs.

\section{Box 2: Potentially more cytoskeletal crosslinker MAPs?}

Several MAPs including MAP2 [164], MAP4 [165], or tau protein [166] have been demonstrated to bind actin. However, the actin-binding domains of MAP2, MAP4 and tau protein have been mapped to the very microtubule binding regions of these proteins [165, 166]. In the case of MAP2 for instance, actin binding is only possible in the absence of tubulin [164], which appears to preclude this MAP from acting as a cytoskeletal crosslinker. Thus, under physiological conditions, an association of those MAPs with the actin cytoskeleton might only happen in cellular compartments with low microtubule content, such as filopodia or leading edges of migrating cells. Strikingly, a recent reconstruction of microtubule and actin networks in vitro has clearly shown that tau protein can connect these two networks with its microtubule-binding repeats. It appears that tau binds to microtubules with at least one of its microtubule-binding repeat, and connects to actin with another of these repeats [167]. Considering that several MAPs, such as MAP2 and MAP4, have similar microtubule-binding repeats, this study provides a new model how MAPs could link microtubule and actin networks only with their microtubule-binding domains. 
A number of MAPs can also interact with intermediate filaments. MAP2 can localize to vimentin in cells, which suggested that it could coordinate intermediate filaments and microtubule networks $[168,169]$. The protein tau has equally been reported to bind vimentin intermediate filaments [170], however, no further functional studies on the role of MAPs in intermediate filament organization and crosstalk with microtubules have been performed. Finally, MAP4 was demonstrated to interact with septins [171], another filamentous network important for a range of cellular functions [172].

Thus, many MAPs have been shown to bind to different cytoskeleton networks, and were thus suggested to act as cytoskeletal crosslinkers. The physiological relevance of these findings, however, remains to be explored.

Figure 1: Timeline of the discovery of mammalian MAPs, and their dependency on the development and availability of experimental approaches. The timeline starts with the first ultrastructural description of microtubules [173], and the identification of their protein building block tubulin [174].

Figure 2: Schematic representation of MAPs scaled to their relative sizes. The approximate localisation of different cytoskeleton-interacting domains is shown (no precise domain boundaries are given due to different representations in the literature). Domain information were obtained from the following references: MACF1, MACF2 [95, 175], MAP1A, MAP1B, MAP8/MAP1S [6, 136], MAP2, MAP4, Tau, MAP6 [136], MAP7, MAP7D1, MAP7D2, MAP7D3 [115], MAP9 [136], MAP10 [40], EML1, EML2, EML3, EML4, EML5, EML6 [176, 177], DCX [6]. The longest annotated human isoforms of each MAP are depicted: MACF1 (XM_006710540), MACF2 (XM_005249315), MAP1A (XM_017022189), MAP1B (NM_005909), MAP8/MAP1S (NM_018174), MAP2 (NM_002374), MAP4 (NM_002375), Tau (NM_005910), MAP6 (NM_033063), MAP7 (NM_001198609), MAP7D1 (BC106053), MAP7D2 (BC136379), MAP7D3 (XM_024452448), MAP9 (AY690636), MAP10 (AB037804), MAP11 (NM_018275), EML1 (NM_004434), EML2 (NM_012155),EML3 (NM_153265), EML4 (NM_019063), EML5 (NM_183387), EML6 (NM_001039753) and DCX (NM_000555). 
Figure 3: Schematic representation of the different ways MAPs can bind to microtubules, and how these modes of binding influences microtubule structure, behaviour, and functions: (A) binding and spacing microtubules with long MAP projection domains, (B) connecting microtubules to membranes, $(\mathrm{C})$ protecting microtubules from severing enzymes, and thus, from disassembly, (D) crosslinking different cytoskeletal elements, (E) controlling the protofilament numbers of microtubules, (F) affecting the binding and motility of motor proteins by either forming a complex with the motor, or by occupying the motor path at the microtubule surface, $(\mathrm{G})$ promoting microtubule bundling, by neutralising acidic tubulin carboxy-terminal tails.

Figure 4: Schematic representation of some emerging mechanisms of MAPs. Depending on their concentration at the microtubules surface, MAPs can form condensates (islands) or undergo phase transition to form liquid droplets. Some MAPs can bind incomplete microtubules, or single protofilaments, thus stabilising such structures, and eventually allowing microtubule repair, or branching. Posttranslational modifications of tubulin could regulate the binding parameters of MAP, thus controlling MAP functions. Vice versa, MAPs might be implicated in controlling microtubule modifications. 


\section{References:}

1. Hirokawa, N. et al. (2009) Kinesin superfamily motor proteins and intracellular transport. Nat Rev Mol Cell Biol 10 (10), 682-96.

2. Bhabha, G. et al. (2016) How Dynein Moves Along Microtubules. Trends Biochem Sci 41 (1), 94-105.

3. McNally, F.J. and Roll-Mecak, A. (2018) Microtubule-severing enzymes: From cellular functions to molecular mechanism. J Cell Biol 217 (12), 4057-4069.

4. Roostalu, J. and Surrey, T. (2017) Microtubule nucleation: beyond the template. Nat Rev Mol Cell Biol 18 (11), 702-710.

5. Akhmanova, A. and Steinmetz, M.O. (2015) Control of microtubule organization and dynamics: two ends in the limelight. Nat Rev Mol Cell Biol 16 (12), 711-26.

6. Ramkumar, A. et al. (2018) ReMAPping the microtubule landscape: How phosphorylation dictates the activities of microtubule-associated proteins. Dev Dyn 247 (1), 138-155.

7. Frost, B. et al. (2015) Connecting the dots between tau dysfunction and neurodegeneration. Trends Cell Biol 25 (1), 46-53.

8. Sloboda, R.D. et al. (1975) Cyclic AMP-dependent endogenous phosphorylation of a microtubule-associated protein. Proc Natl Acad Sci U S A 72 (1), 177-81.

9. Borisy, G.G. et al. (1974) Microtubule assembly in vitro. Fed Proc 33 (2), 167-74.

10. Weingarten, M.D. et al. (1975) A protein factor essential for microtubule assembly. Proc Natl Acad Sci U S A 72 (5), 1858-1862.

11. Cleveland, D.W. et al. (1977) Purification of tau, a microtubule-associated protein that induces assembly of microtubules from purified tubulin. J Mol Biol 116 (2), 207-225.

12. Cleveland, D.W. et al. (1977) Physical and chemical properties of purified tau factor and the role of tau in microtubule assembly. J Mol Biol 116 (2), 227-247.

13. Herzog, W. and Weber, K. (1978) Fractionation of brain microtubule-associated proteins. Isolation of two different proteins which stimulate tubulin polymerization in vitro. Eur $\mathbf{J}$ Biochem 92 (1), 1-8.

14. Bulinski, J.C. and Borisy, G.G. (1979) Self-assembly of microtubules in extracts of cultured HeLa cells and the identification of HeLa microtubule-associated proteins. Proc Natl Acad Sci U S A 76 (1), 293-7.

15. Bulinski, J.C. and Borisy, G.G. (1980) Microtubule-associated proteins from cultured HeLa cells. Analysis of molecular properties and effects on microtubule polymerization. J Biol Chem 255 (23), 11570-6.

16. Parysek, L.M. et al. (1984) MAP 4: occurrence in mouse tissues. J Cell Biol 99 (4 Pt 1), 1309-15.

17. Masson, D. and Kreis, T.E. (1993) Identification and molecular characterization of EMAP-115, a novel microtubule-associated protein predominantly expressed in epithelial cells. J Cell Biol 123 (2), 357-71.

18. Faire, K. et al. (1999) E-MAP-115 (ensconsin) associates dynamically with microtubules in vivo and is not a physiological modulator of microtubule dynamics. J Cell Sci 112 ( Pt 23), 4243-55.

19. Webb, B.C. and Wilson, L. (1980) Cold-stable microtubules from brain. Biochemistry 19 (9), 1993-2001. 
20. Job, D. et al. (1982) Recycling of cold-stable microtubules: evidence that cold stability is due to substoichiometric polymer blocks. Biochemistry 21 (3), 509-15.

21. Margolis, R.L. et al. (1986) Purification and assay of a 145-kDa protein (STOP145) with microtubule- stabilizing and motility behavior. Proc Natl Acad Sci U S A 83 (3), 639-43.

22. Black, M.M. and Kurdyla, J.T. (1983) Microtubule-associated proteins of neurons. J Cell Biol 97 (4), 1020-8.

23. Murphy, D.B. et al. (1977) Identity and polymerization-stimulatory activity of the nontubulin proteins associated with microtubules. Biochemistry 16 (12), 2598-605.

24. Huber, G. et al. (1985) MAP3: characterization of a novel microtubule-associated protein. J Cell Biol 100 (2), 496-507.

25. Riederer, B. et al. (1986) MAP5: a novel brain microtubule-associated protein under strong developmental regulation. J Neurocytol 15 (6), 763-75.

26. Paschal, B.M. and Vallee, R.B. (1987) Retrograde transport by the microtubule-associated protein MAP 1C. Nature 330 (6144), 181-3.

27. Paschal, B.M. et al. (1987) MAP 1C is a microtubule-activated ATPase which translocates microtubules in vitro and has dynein-like properties. J Cell Biol 105 (3), 1273-82.

28. Garner, C.C. et al. (1990) Molecular cloning of microtubule-associated protein 1 (MAP1A) and microtubule-associated protein 5 (MAP1B): identification of distinct genes and their differential expression in developing brain. J Neurochem 55 (1), 146-54.

29. Papandrikopoulou, A. et al. (1989) Embryonic MAP2 lacks the cross-linking sidearm sequences and dendritic targeting signal of adult MAP2. Nature 340 (6235), 650-2.

30. Kindler, S. et al. (1990) Molecular structure of microtubule-associated protein $2 \mathrm{~b}$ and $2 \mathrm{c}$ from rat brain. J Biol Chem 265 (32), 19679-84.

31. Kobayashi, N. et al. (2000) Molecular characterization reveals identity of microtubuleassociated proteins MAP3 and MAP4. Biochem Biophys Res Commun 268 (2), 306-9.

32. Goedert, M. et al. (1989) Multiple isoforms of human microtubule-associated protein tau: sequences and localization in neurofibrillary tangles of Alzheimer's disease. Neuron 3 (4), 519-526.

33. Himmler, A. (1989) Structure of the bovine tau gene: alternatively spliced transcripts generate a protein family. Mol Cell Biol 9 (4), 1389-96.

34. Goedert, M. et al. (1988) Cloning and sequencing of the cDNA encoding a core protein of the paired helical filament of Alzheimer disease: identification as the microtubuleassociated protein tau. Proc Natl Acad Sci U S A 85 (11), 4051-5.

35. Vallee, R.B. and Bloom, G.S. (1983) Isolation of sea urchin egg microtubules with taxol and identification of mitotic spindle microtubule-associated proteins with monoclonal antibodies. Proc Natl Acad Sci U S A 80 (20), 6259-63.

36. Keller, T.C., 3rd and Rebhun, L.I. (1982) Strongylocentrotus purpuratus spindle tubulin. I. Characteristics of its polymerization and depolymerization in vitro. J Cell Biol 93 (3), 788-96.

37. Lepley, D.M. et al. (1999) Sequence and expression patterns of a human EMAP-related protein-2 (HuEMAP-2). Gene 237 (2), 343-9.

38. Eudy, J.D. et al. (1997) Isolation of a novel human homologue of the gene coding for echinoderm microtubule-associated protein (EMAP) from the Usher syndrome type 1a locus at 14q32. Genomics 43 (1), 104-6. 
39. Manabe, R.i. et al. (2002) Identification of a Novel Microtubule-Associated Protein that Regulates Microtubule Organization and Cytokinesis by Using a GFP-Screening Strategy. Curr Biol 12 (22), 1946-1951.

40. Fong, K.-W. et al. (2013) MTR120/KIAA1383, a novel microtubule-associated protein, promotes microtubule stability and ensures cytokinesis. J Cell Sci 126 (Pt 3), 825-37.

41. Wong, E.Y.M. et al. (2004) Identification and characterization of human VCY2interacting protein: VCY2IP-1, a microtubule-associated protein-like protein. Biol Reprod 70 (3), 775-84.

42. Orban-Nemeth, Z. et al. (2005) Microtubule-associated protein 1S, a short and ubiquitously expressed member of the microtubule-associated protein 1 family. J Biol Chem 280 (3), 2257-65.

43. Saffin, J.-M. et al. (2005) ASAP, a human microtubule-associated protein required for bipolar spindle assembly and cytokinesis. Proc Natl Acad Sci U S A 102 (32), 11302-7.

44. Perez, Y. et al. (2019) Mutations in the microtubule-associated protein MAP11 (C7orf43) cause microcephaly in humans and zebrafish. Brain 142 (3), 574-585.

45. Gache, V. et al. (2010) Xenopus meiotic microtubule-associated interactome. PLoS One 5 (2), e9248.

46. Murphy, D.B. and Borisy, G.G. (1975) Association of high-molecular-weight proteins with microtubules and their role in microtubule assembly in vitro. Proc Natl Acad Sci U S A 72 (7), 2696-700.

47. Sloboda, R.D. et al. (1976) Microtubule-associated proteins and the stimulation of tubulin assembly in vitro. Biochemistry 15 (20), 4497-505.

48. Barlow, S. et al. (1994) Stable expression of heterologous microtubule-associated proteins (MAPs) in Chinese hamster ovary cells: evidence for differing roles of MAPs in microtubule organization. J Cell Biol 126 (4), 1017-29.

49. Kanai, Y. et al. (1989) Expression of multiple tau isoforms and microtubule bundle formation in fibroblasts transfected with a single tau cDNA. J Cell Biol 109 (3), 1173-84.

50. Lewis, S.A. et al. (1989) Organization of microtubules in dendrites and axons is determined by a short hydrophobic zipper in microtubule-associated proteins MAP2 and tau. Nature 342 (6249), 498-505.

51. Chapin, S.J. et al. (1991) Microtubule bundling in cells. Nature 349 (6304), 24.

52. Scott, C.W. et al. (1992) Tau protein induces bundling of microtubules in vitro: comparison of different tau isoforms and a tau protein fragment. J Neurosci Res 33 (1), 19-29.

53. Wang, Q. et al. (2014) Structural basis for the extended CAP-Gly domains of p150(glued) binding to microtubules and the implication for tubulin dynamics. Proc Natl Acad Sci U S A 111 (31), 11347-52.

54. Sackett, D.L. et al. (1985) Tubulin subunit carboxyl termini determine polymerization efficiency. J Biol Chem 260 (1), 43-5.

55. Nguyen, H.L. et al. (1997) Overexpression of full- or partial-length MAP4 stabilizes microtubules and alters cell growth. J Cell Sci 110 ( Pt 2), 281-94.

56. Iida, J. et al. (2002) The projection domain of MAP4 suppresses the microtubule-bundling activity of the microtubule-binding domain. J Mol Biol 320 (1), 97-106.

57. Chen, J. et al. (1992) Projection domains of MAP2 and tau determine spacings between microtubules in dendrites and axons. Nature 360 (6405), 674-7. 
58. Harada, A. et al. (1994) Altered microtubule organization in small-calibre axons of mice lacking tau protein. Nature 369 (6480), 488-91.

59. Georgieff, I.S. et al. (1993) Expression of high molecular weight tau in the central and peripheral nervous systems. J Cell Sci 105 (Pt 3), 729-37.

60. Kanai, Y. et al. (1992) Microtubule bundling by tau proteins in vivo: analysis of functional domains. Embo J 11 (11), 3953-61.

61. Takemura, R. et al. (1992) Increased microtubule stability and alpha tubulin acetylation in cells transfected with microtubule-associated proteins MAP1B, MAP2 or tau. J Cell Sci 103 (Pt 4), 953-64.

62. Nogales, E. (2016) The development of cryo-EM into a mainstream structural biology technique. Nat Methods 13 (1), 24-7.

63. Kellogg, E.H. et al. (2018) Near-atomic model of microtubule-tau interactions. Science 360 (6394), 1242-1246.

64. Serrano, L. et al. (1985) Localization of the tubulin binding site for tau protein. Eur J Biochem 153 (3), 595-600.

65. Boucher, D. et al. (1994) Polyglutamylation of tubulin as a progressive regulator of in vitro interactions between the microtubule-associated protein Tau and tubulin. Biochemistry 33 (41), 12471-12477.

66. Shigematsu, H. et al. (2018) Structural insight into microtubule stabilization and kinesin inhibition by Tau family MAPs. J Cell Biol 217 (12), 4155-4163.

67. Fourniol, F.J. et al. (2010) Template-free 13-protofilament microtubule-MAP assembly visualized at 8 A resolution. J Cell Biol 191 (3), 463-70.

68. Janning, D. et al. (2014) Single-molecule tracking of tau reveals fast kiss-and-hop interaction with microtubules in living neurons. Mol Biol Cell 25 (22), 3541-51.

69. Qiang, L. et al. (2018) Tau Does Not Stabilize Axonal Microtubules but Rather Enables Them to Have Long Labile Domains. Curr Biol 28 (13), 2181-2189 e4.

70. Baas, P.W. and Qiang, L. (2019) Tau: It's Not What You Think. Trends Cell Biol 29 (6), 452-461.

71. Gonzalez-Billault, C. et al. (2001) Evidence for the role of MAP1B in axon formation. Mol Biol Cell 12 (7), 2087-98.

72. Utreras, E. et al. (2008) Microtubule-associated protein 1B interaction with tubulin tyrosine ligase contributes to the control of microtubule tyrosination. Dev Neurosci 30 (13), 200-10.

73. Palazzo, A.F. and Gundersen, G.G. (2002) Microtubule-actin cross-talk at focal adhesions. Sci STKE 2002 (139), pe31.

74. Vitriol, E.A. and Zheng, J.Q. (2012) Growth cone travel in space and time: the cellular ensemble of cytoskeleton, adhesion, and membrane. Neuron 73 (6), 1068-81.

75. Bernier, G. et al. (1996) Cloning and characterization of mouse ACF7, a novel member of the dystonin subfamily of actin binding proteins. Genomics 38 (1), 19-29.

76. Sawamura, D. et al. (1990) Bullous pemphigoid antigen (BPAG1): cDNA cloning and mapping of the gene to the short arm of human chromosome 6. Genomics 8 (4), 722-6.

77. Bernier, G. et al. (1995) Dystonin expression in the developing nervous system predominates in the neurons that degenerate in dystonia musculorum mutant mice. Mol Cell Neurosci 6 (6), 509-20. 
78. Leung, C.L. et al. (1999) Microtubule actin cross-linking factor (MACF): a hybrid of dystonin and dystrophin that can interact with the actin and microtubule cytoskeletons. $\mathrm{J}$ Cell Biol 147 (6), 1275-86.

79. Karakesisoglou, I. et al. (2000) An epidermal plakin that integrates actin and microtubule networks at cellular junctions. J Cell Biol 149 (1), 195-208.

80. Yang, Y. et al. (1996) An essential cytoskeletal linker protein connecting actin microfilaments to intermediate filaments. Cell 86 (4), 655-65.

81. Leung, C.L. et al. (1999) The intermediate filament protein peripherin is the specific interaction partner of mouse BPAG1-n (dystonin) in neurons. J Cell Biol 144 (3), 435-46.

82. Sun, D. et al. (2001) Characterization of the microtubule binding domain of microtubule actin crosslinking factor (MACF): identification of a novel group of microtubule associated proteins. J Cell Sci 114 (Pt 1), 161-172.

83. Levine, B.A. et al. (1990) The interaction of actin with dystrophin. FEBS Lett 263 (1), 159-62.

84. Prins, K.W. et al. (2009) Dystrophin is a microtubule-associated protein. J Cell Biol 186 (3), 363-9.

85. Preciado Lopez, M. et al. (2014) Actin-microtubule coordination at growing microtubule ends. Nat Commun 5, 4778.

86. Wu, X. et al. (2008) ACF7 regulates cytoskeletal-focal adhesion dynamics and migration and has ATPase activity. Cell 135 (1), 137-48.

87. $\mathrm{Wu}, \mathrm{X}$. et al. (2011) Skin stem cells orchestrate directional migration by regulating microtubule-ACF7 connections through GSK3beta. Cell 144 (3), 341-52.

88. Goryunov, D. et al. (2010) Nervous-tissue-specific elimination of microtubule-actin crosslinking factor 1a results in multiple developmental defects in the mouse brain. Mol Cell Neurosci 44 (1), 1-14.

89. Ka, M. et al. (2014) MACF1 regulates the migration of pyramidal neurons via microtubule dynamics and GSK-3 signaling. Dev Biol 395 (1), 4-18.

90. Sanchez-Soriano, N. et al. (2009) Mouse ACF7 and drosophila short stop modulate filopodia formation and microtubule organisation during neuronal growth. J Cell Sci 122 (Pt 14), 2534-42.

91. Ka, M. and Kim, W.-Y. (2016) Microtubule-Actin Crosslinking Factor 1 Is Required for Dendritic Arborization and Axon Outgrowth in the Developing Brain. Mol Neurobiol 53 (9), 6018-6032.

92. Dalpe, G. et al. (1998) Dystonin is essential for maintaining neuronal cytoskeleton organization. Mol Cell Neurosci 10 (5-6), 243-57.

93. Kunzli, K. et al. (2016) One gene but different proteins and diseases: the complexity of dystonin and bullous pemphigoid antigen 1. Exp Dermatol 25 (1), 10-6.

94. Jefferson, J.J. et al. (2004) Plakins: goliaths that link cell junctions and the cytoskeleton. Nat Rev Mol Cell Biol 5 (7), 542-53.

95. Zhang, J. et al. (2017) Spectraplakin family proteins - cytoskeletal crosslinkers with versatile roles. J Cell Sci 130 (15), 2447-2457.

96. Houseweart, M.K. and Cleveland, D.W. (1999) Cytoskeletal linkers: new MAPs for old destinations. Curr Biol 9 (22), R864-866. 
97. Pedrotti, B. et al. (1994) Microtubule associated protein MAP1A is an actin-binding and crosslinking protein. Cell Motil Cytoskeleton 29 (2), 110-6.

98. Tögel, M. et al. (1998) Novel features of the light chain of microtubule-associated protein MAP1B: microtubule stabilization, self interaction, actin filament binding, and regulation by the heavy chain. J Cell Biol 143 (3), 695-707.

99. Halpain, S. and Dehmelt, L. (2006) The MAP1 family of microtubule-associated proteins. Genome Biol 7 (6), 224.

100. Montenegro-Venegas, C. et al. (2010) MAP1B regulates axonal development by modulating Rho-GTPase Rac1 activity. Mol Biol Cell 21 (20), 3518-28.

101. Wang, Y. and Mandelkow, E. (2016) Tau in physiology and pathology. Nat Rev Neurosci 17 (1), 22-35.

102. Ishihara, T. et al. (1999) Age-dependent emergence and progression of a tauopathy in transgenic mice overexpressing the shortest human tau isoform. Neuron 24 (3), 751-62.

103. Mandelkow, E.-M. et al. (2003) Clogging of axons by tau, inhibition of axonal traffic and starvation of synapses. Neurobiol Aging 24 (8), 1079-85.

104. Ebneth, A. et al. (1998) Overexpression of tau protein inhibits kinesin-dependent trafficking of vesicles, mitochondria, and endoplasmic reticulum: implications for Alzheimer's disease. J Cell Biol 143 (3), 777-94.

105. Vossel, K.A. et al. (2010) Tau reduction prevents Abeta-induced defects in axonal transport. Science 330 (6001), 198.

106. Shahpasand, K. et al. (2012) Regulation of mitochondrial transport and inter-microtubule spacing by tau phosphorylation at the sites hyperphosphorylated in Alzheimer's disease. J Neurosci 32 (7), 2430-41.

107. Vershinin, M. et al. (2007) Multiple-motor based transport and its regulation by Tau. Proc Natl Acad Sci U S A 104 (1), 87-92.

108. Dixit, R. et al. (2008) Differential regulation of dynein and kinesin motor proteins by tau. Science 319 (5866), 1086-9.

109. Jimenez-Mateos, E.-M. et al. (2006) Role of MAP1B in axonal retrograde transport of mitochondria. Biochem J 397 (1), 53-9.

110. Mandelkow, E.-M. et al. (2004) MARK/PAR1 kinase is a regulator of microtubuledependent transport in axons. J Cell Biol 167 (1), 99-110.

111. Semenova, I. et al. (2014) Regulation of microtubule-based transport by MAP4. Mol Biol Cell 25 (20), 3119-32.

112. Daoust, A. et al. (2014) Neuronal transport defects of the MAP6 KO mouse - a model of schizophrenia - and alleviation by Epothilone D treatment, as observed using MEMRI. Neuroimage 96, 133-42.

113. Monroy, B.Y. et al. (2018) Competition between microtubule-associated proteins directs motor transport. Nat Commun 9 (1), 1487.

114. Tymanskyj, S.R. et al. (2018) MAP7 regulates axon morphogenesis by recruiting kinesin-1 to microtubules and modulating organelle transport. Elife 7.

115. Hooikaas, P.J. et al. (2019) MAP7 family proteins regulate kinesin-1 recruitment and activation. J Cell Biol 218 (4), 1298-1318.

116. Metivier, M. et al. (2018) Mechanisms of Kinesin-1 activation by Ensconsin/MAP7 in vivo. bioRxiv, 325035 . 
117. Gumy, L.F. et al. (2017) MAP2 Defines a Pre-axonal Filtering Zone to Regulate KIF1versus KIF5-Dependent Cargo Transport in Sensory Neurons. Neuron 94 (2), 347-362 e7.

118. McNally, K.P. et al. (2002) Katanin-mediated microtubule severing can be regulated by multiple mechanisms. Cell Motil Cytoskeleton 53 (4), 337-49.

119. Qiang, L. et al. (2006) Tau protects microtubules in the axon from severing by katanin. J Neurosci 26 (12), 3120-9.

120. Siahaan, V. et al. (2018) Kinetically distinct phases of tau on microtubules regulate kinesin motors and severing enzymes. bioRxiv, 424374.

121. Tan, R. et al. (2018) Microtubules Gate Tau Condensation to Spatially Regulate Microtubule Functions. bioRxiv, 423376.

122. Barlan, K. et al. (2013) The microtubule-binding protein ensconsin is an essential cofactor of kinesin-1. Curr Biol 23 (4), 317-22.

123. Yu, W. et al. (2008) The Microtubule-severing Proteins Spastin and Katanin Participate Differently in the Formation of Axonal Branches. Mol Biol Cell 19 (4), 1485-98.

124. Tymanskyj, S.R. et al. (2017) MAP7 Regulates Axon Collateral Branch Development in Dorsal Root Ganglion Neurons. J Neurosci 37 (6), 1648-1661.

125. Dehmelt, L. and Halpain, S. (2005) The MAP2/Tau family of microtubule-associated proteins. Genome Biol 6 (1), 204.

126. Villarroel-Campos, D. and Gonzalez-Billault, C. (2014) The MAP1B case: an old MAP that is new again. Dev Neurobiol 74 (10), 953-71.

127. Obar, R.A. et al. (1989) The RII subunit of cAMP-dependent protein kinase binds to a common amino-terminal domain in microtubule-associated proteins $2 \mathrm{~A}, 2 \mathrm{~B}$, and $2 \mathrm{C}$. Neuron 3 (5), 639-45.

128. Lim, R.W. and Halpain, S. (2000) Regulated association of microtubule-associated protein 2 (MAP2) with Src and Grb2: evidence for MAP2 as a scaffolding protein. J Biol Chem 275 (27), 20578-87.

129. Zamora-Leon, S.P. et al. (2001) Binding of Fyn to MAP-2c through an SH3 binding domain. Regulation of the interaction by ERK2. J Biol Chem 276 (43), 39950-8.

130. Harada, A. et al. (2002) MAP2 is required for dendrite elongation, PKA anchoring in dendrites, and proper PKA signal transduction. J Cell Biol 158 (3), 541-9.

131. Oury, J. et al. (2019) MACF1 links Rapsyn to microtubule- and actin-binding proteins to maintain neuromuscular synapses. J Cell Biol 218 (5), 1686-1705.

132. Brandt, R. et al. (1995) Interaction of tau with the neural plasma membrane mediated by tau's amino-terminal projection domain. J Cell Biol 131 (5), 1327-1340.

133. Kakinuma, T. et al. (2004) Interaction between p230 and MACF1 is associated with transport of a glycosyl phosphatidyl inositol-anchored protein from the Golgi to the cell periphery. Exp Cell Res 298 (2), 388-98.

134. Villarroel-Campos, D. et al. (2016) Rab35 Functions in Axon Elongation Are Regulated by P53-Related Protein Kinase in a Mechanism That Involves Rab35 Protein Degradation and the Microtubule-Associated Protein 1B. J Neurosci 36 (27), 7298-313.

135. Fernandez, J. et al. (2015) Microtubule-associated proteins 1 (MAP1) promote human immunodeficiency virus type I (HIV-1) intracytoplasmic routing to the nucleus. J Biol Chem 290 (8), 4631-46. 
136. Tortosa, E. et al. (2016) Microtubule Organization and Microtubule-Associated Proteins (MAPs). In Dendrites: Development and Disease (Emoto, K. et al. eds), pp. 31-75, Springer Japan.

137. Lei, P. et al. (2012) Tau deficiency induces parkinsonism with dementia by impairing APP-mediated iron export. Nat Med 18 (2), 291-5.

138. Meixner, A. et al. (2000) MAP1B is required for axon guidance and Is involved in the development of the central and peripheral nervous system [In Process Citation]. J Cell Biol 151 (6), 1169-1178.

139. Bouquet, C. et al. (2004) Microtubule-associated protein 1B controls directionality of growth cone migration and axonal branching in regeneration of adult dorsal root ganglia neurons. J Neurosci 24 (32), 7204-13.

140. Bodaleo, F.J. et al. (2016) Microtubule-associated protein 1B (MAP1B)-deficient neurons show structural presynaptic deficiencies in vitro and altered presynaptic physiology. Sci Rep 6, 30069.

141. Liu, Y. et al. (2015) Mutations in the Microtubule-Associated Protein 1A (Map1a) Gene Cause Purkinje Cell Degeneration. J Neurosci 35 (11), 4587-98.

142. Andrieux, A. et al. (2002) The suppression of brain cold-stable microtubules in mice induces synaptic defects associated with neuroleptic-sensitive behavioral disorders. Genes Dev 16 (18), 2350-2364.

143. Powell, K.J. et al. (2007) Cognitive impairments in the STOP null mouse model of schizophrenia. Behav Neurosci 121 (5), 826-35.

144. Goedert, M. and Jakes, R. (2005) Mutations causing neurodegenerative tauopathies. Biochim Biophys Acta 1739 (2-3), 240-50.

145. Kovacs, G.G. (2017) Tauopathies. Handb Clin Neurol 145, 355-368.

146. Magiera, M.M. et al. (2018) Excessive tubulin polyglutamylation causes neurodegeneration and perturbs neuronal transport. EMBO J 37 (23), e100440.

147. Shashi, V. et al. (2018) Loss of tubulin deglutamylase CCP1 causes infantile-onset neurodegeneration. EMBO J 37 (23), e100540.

148. van Beuningen, S.F.B. et al. (2015) TRIM46 Controls Neuronal Polarity and Axon Specification by Driving the Formation of Parallel Microtubule Arrays. Neuron 88 (6), 1208-26.

149. Bieling, P. et al. (2010) A minimal midzone protein module controls formation and length of antiparallel microtubule overlaps. Cell 142 (3), 420-32.

150. Subramanian, R. et al. (2010) Insights into antiparallel microtubule crosslinking by PRC1, a conserved nonmotor microtubule binding protein. Cell 142 (3), 433-43.

151. Janke, C. (2014) The tubulin code: Molecular components, readout mechanisms, and functions. J Cell Biol 206 (4), 461-472.

152. Zwicker, D. et al. (2014) Centrosomes are autocatalytic droplets of pericentriolar material organized by centrioles. Proc Natl Acad Sci U S A 111 (26), E2636-45.

153. Hernandez-Vega, A. et al. (2017) Local Nucleation of Microtubule Bundles through Tubulin Concentration into a Condensed Tau Phase. Cell Rep 20 (10), 2304-2312.

154. Chretien, D. et al. (1992) Lattice defects in microtubules: protofilament numbers vary within individual microtubules. J Cell Biol 117 (5), 1031-40. 
155. Basnet, N. et al. (2018) Direct induction of microtubule branching by microtubule nucleation factor SSNA1. Nat Cell Biol 20 (10), 1172-1180.

156. Aumeier, C. et al. (2016) Self-repair promotes microtubule rescue. Nat Cell Biol 18 (10), 1054-64.

157. Aher, A. et al. (2018) CLASP Suppresses Microtubule Catastrophes through a Single TOG Domain. Dev Cell 46 (1), 40-58 e8.

158. Dumont, E.L.P. et al. (2015) Molecular wear of microtubules propelled by surfaceadhered kinesins. Nat Nanotechnol 10 (2), 166-9.

159. Triclin, S. et al. (2018) Self-repair protects microtubules from their destruction by molecular motors. bioRxiv, 499020.

160. Ichikawa, M. and Bui, K.H. (2018) Microtubule Inner Proteins: A Meshwork of Luminal Proteins Stabilizing the Doublet Microtubule. Bioessays 40 (3).

161. Schmidt-Cernohorska, M. et al. (2019) Flagellar microtubule doublet assembly in vitro reveals a regulatory role of tubulin C-terminal tails. Science 363 (6424), 285-288.

162. Yu, N. et al. (2016) Isolation of Functional Tubulin Dimers and of Tubulin-Associated Proteins from Mammalian Cells. Curr Biol 26 (13), 1728-36.

163. Jourdain, L. et al. (1997) Stathmin: a tubulin-sequestering protein which forms a ternary T2S complex with two tubulin molecules. Biochemistry 36 (36), 10817-21.

164. Sattilaro, R.F. (1986) Interaction of microtubule-associated protein 2 with actin filaments. Biochemistry 25 (8), 2003-9.

165. Matsushima, K. et al. (2012) Microtubule-associated protein 4 binds to actin filaments and modulates their properties. J Biochem 151 (1), 99-108.

166. Correas, I. et al. (1990) The tubulin-binding sequence of brain microtubule-associated proteins, tau and MAP-2, is also involved in actin binding. Biochem J 269 (1), 61-4.

167. Elie, A. et al. (2015) Tau co-organizes dynamic microtubule and actin networks. Sci Rep $5,9964$.

168. Bloom, G.S. and Vallee, R.B. (1983) Association of microtubule-associated protein 2 (MAP 2) with microtubules and intermediate filaments in cultured brain cells. J Cell Biol 96 (6), 1523-31.

169. Vallee, R.B. et al. (1984) Microtubule-associated proteins: subunits of the cytomatrix. J Cell Biol 99 (1 Pt 2), 38s-44s.

170. Capote, C. and Maccioni, R.B. (1998) The association of tau-like proteins with vimentin filaments in cultured cells. Exp Cell Res 239 (2), 202-13.

171. Kremer, B.E. et al. (2005) Mammalian septins regulate microtubule stability through interaction with the microtubule-binding protein MAP4. Mol Biol Cell 16 (10), 4648-59.

172. Mostowy, S. and Cossart, P. (2012) Septins: the fourth component of the cytoskeleton. Nat Rev Mol Cell Biol 13 (3), 183-94.

173. Pease, D.C. (1963) The Ultrastructure of Flagellar Fibrils. J Cell Biol 18, 313-26.

174. Borisy, G.G. and Taylor, E.W. (1967) The mechanism of action of colchicine. Binding of colchincine-3H to cellular protein. J Cell Biol 34 (2), 525-33.

175. Liem, R.K.H. (2016) Cytoskeletal Integrators: The Spectrin Superfamily. Cold Spring Harb Perspect Biol 8 (10), 8:a018259.

176. Fry, A.M. et al. (2016) EML proteins in microtubule regulation and human disease. Biochem Soc Trans 44 (5), 1281-1288. 
177. Richards, M.W. et al. (2015) Microtubule association of EML proteins and the EML4ALK variant 3 oncoprotein require an N-terminal trimerization domain. Biochem J 467 (3), 529-36. 


\section{Timeline: a brief history of MAP discoveries}

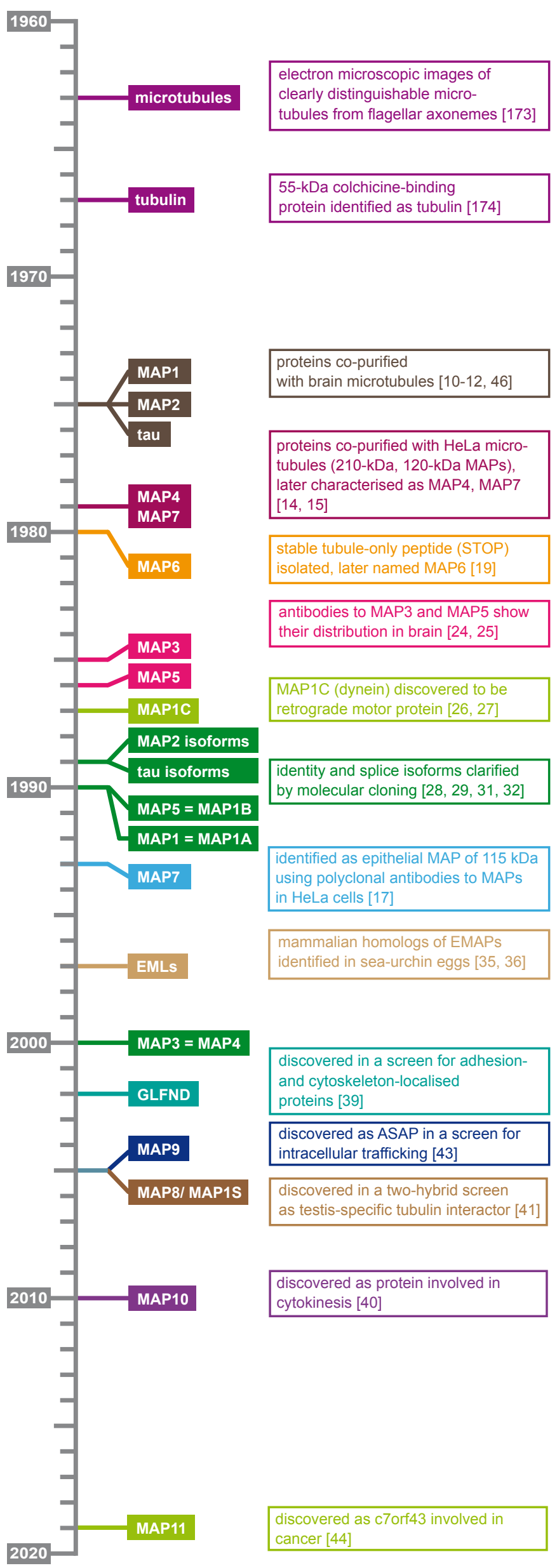




\section{Relative size and domain organisation of MAPs}

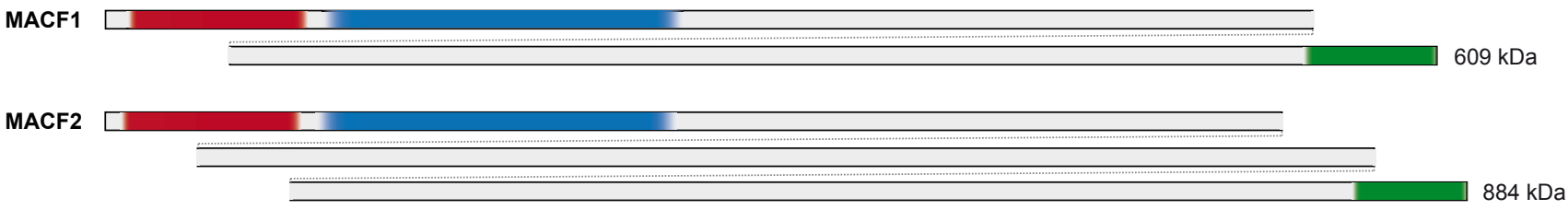

MAP1A

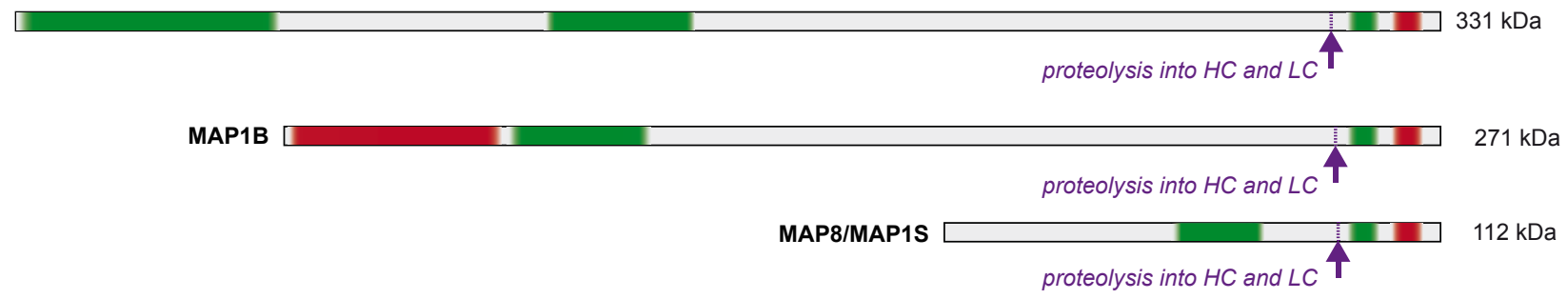

MAP2

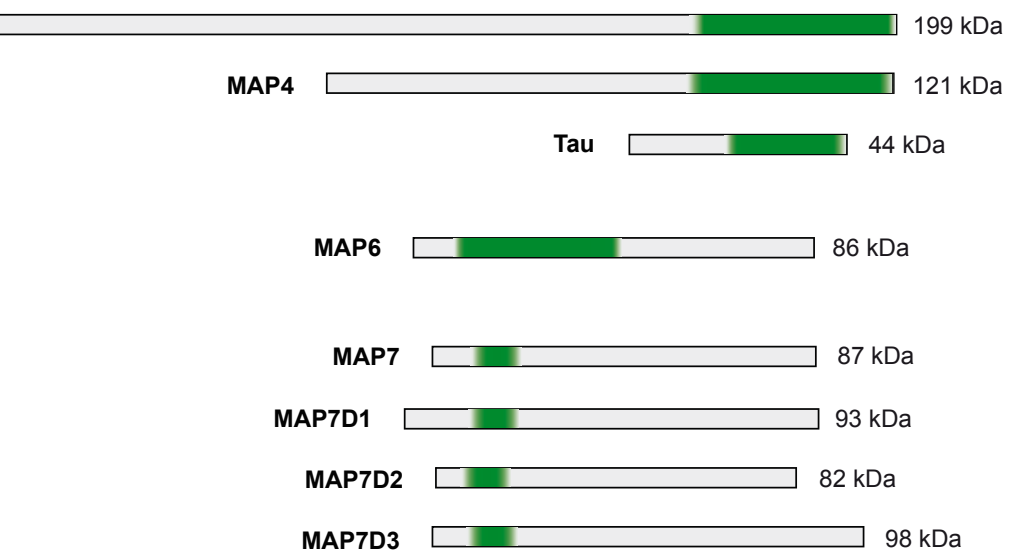

MAP9 $\square 74 \mathrm{kDa}$

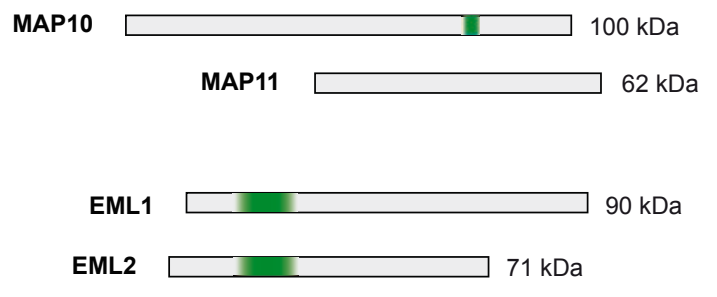

approximate domain distribution

microtubule binding

actin interactions

intermediate-filament interactions

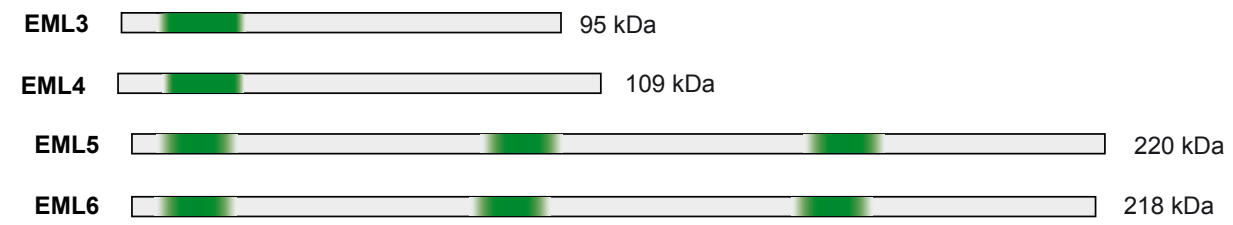

DCX $49 \mathrm{kDa}$ 


\section{Organisation of microtubule cytoskeleton by MAPs}

\section{A}

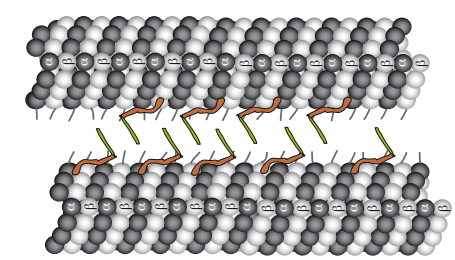

B membune

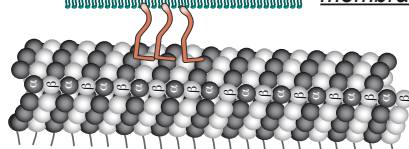
MAPs connect microtubules and membranes
C

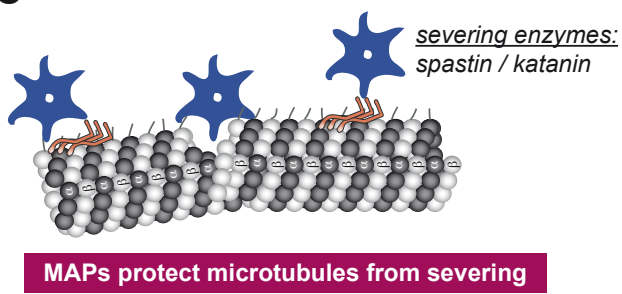

MAP projection domains control microtubule spacing

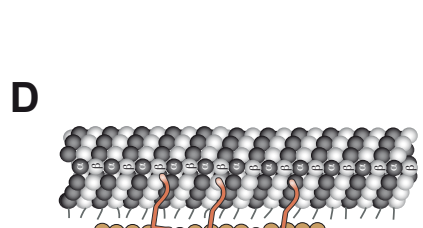

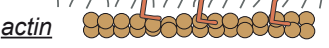

\begin{tabular}{|c|c|}
\hline 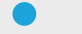 & nucleus \\
\hline \ & MAP \\
\hline & centrioles \\
\hline & MAP7 \\
\hline & $\begin{array}{l}\text { MAPs with } \\
\text { longer projec- } \\
\text { tion domain }\end{array}$ \\
\hline$\omega^{+}$ & $\begin{array}{l}\text { microtubule } \\
\text { plus end }\end{array}$ \\
\hline
\end{tabular}

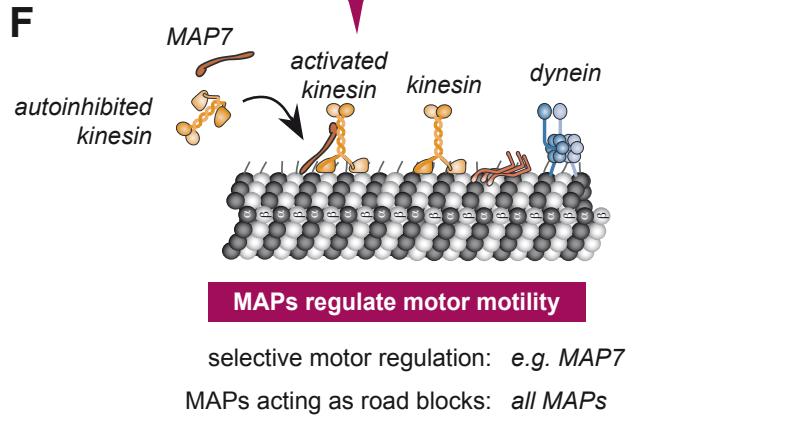

E

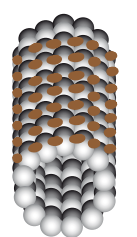

e.g. doublecortin protofilament numbers

\section{G}
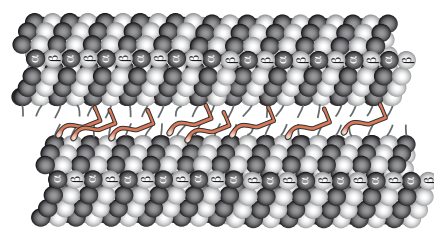

MAPs bundle microtubules

anti-parallel bundling:

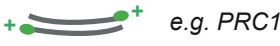

parallel bundling:

${ }^{+}$e.g. TRIM46 


\section{Emerging mechanisms of MAPs}

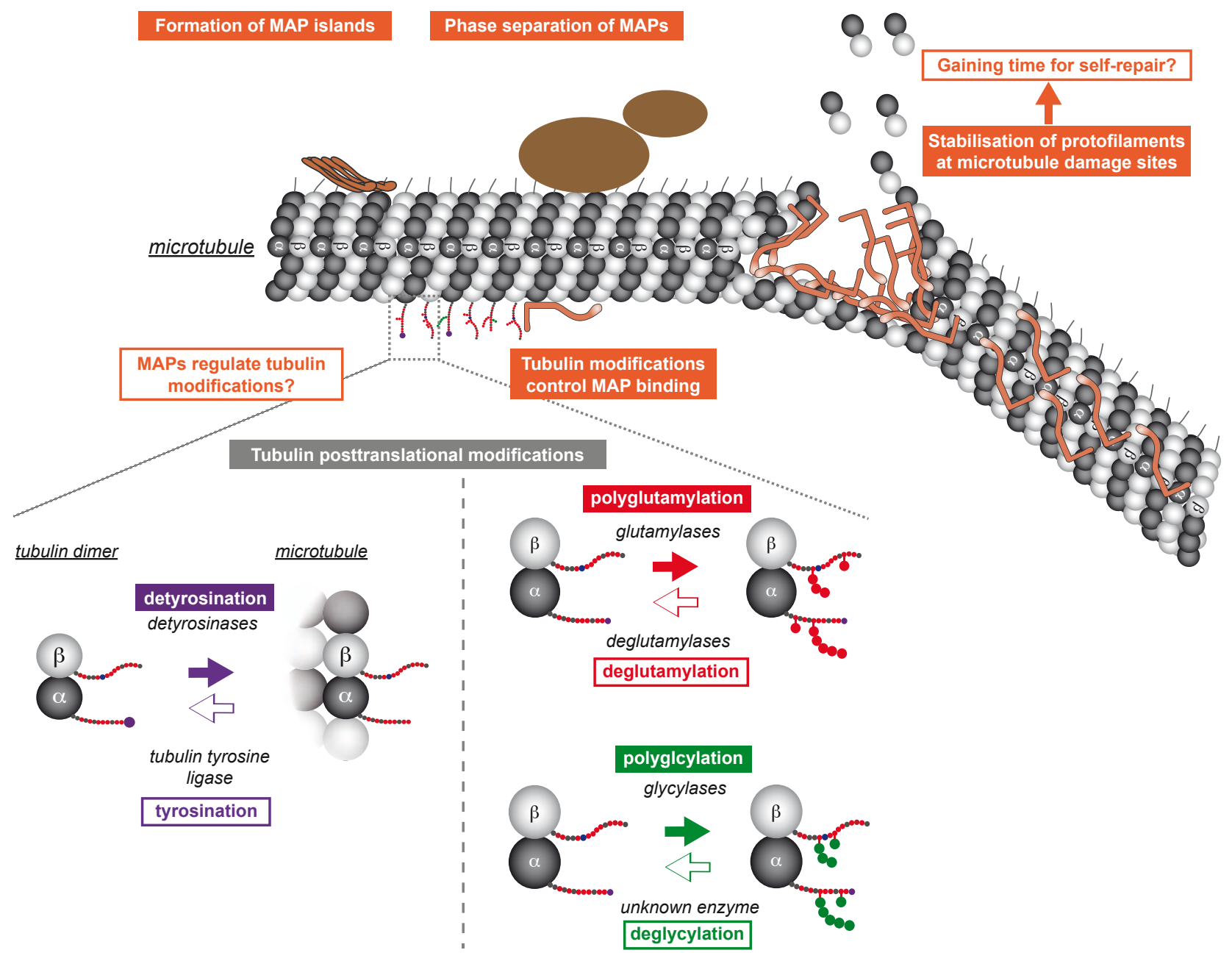

